\title{
MOLECULAR DYNAMICS SIMULATION OF DISSOCIATION KINETICS
}

\author{
AIAA Paper 2000-0213 \\ Presented at the AIAA Aersospace \\ Sciences Meeting
}

Andrew L. Kantor, Lyle N. Long, and Michael M. Micci

Department of Aerospace Engineering

The Pennsylvania State University

University Park, PA 16802 


\title{
MOLECULAR DYNAMICS SIMULATION OF DISSOCIATION KINETICS
}

\author{
Andrew L. Kantor, Lyle N. Long, and Michael M. Micci \\ Department of Aerospace Engineering \\ The Pennsylvania State University \\ University Park, PA 16802
}

\begin{abstract}
$\underline{\text { Abstract }}$
The vibrational energy distribution and the degree of dissociation within a system of hydrogen and oxygen molecules was modeled using molecular dynamics (MD). The first step in this process was to model the atomic and molecular interactions. Since hydrogen and oxygen form diatomic molecules, vibration is the only intramolecular force that must be computed. The Morse potential is used to perform this calculation. Atomic interactions outside the molecule are modeled using the Lennard-Jones potential. The vibrational energy level distribution of this model demonstrated excellent agreement with the Boltzmann distribution. In this molecular dynamics simulation, dissociation occurs when the potential energy between two vibrating atoms exceeds a critical value. Recombination is also possible between two previously dissociated atoms by the reverse mechanism. This process enables a system to start in a state of molecules and proceed to an equilibrated state of atoms and molecules. The molecular dynamics simulation accurately modeled both the rate of dissociation and the ratio of species at equilibrium. Finally, a system of both hydrogen and oxygen was simulated. The results of this simulation were compared to the CET code and showed excellent agreement. This investigation demonstrated that simple chemical reactions in relatively large systems can be modeled using molecular dynamics.
\end{abstract}

\section{$\underline{\text { Nomenclature }}$}

$\mathrm{a}=$ acceleration vector

$\mathrm{C}_{\mathrm{f}}=$ rate constant

$\mathrm{D}=$ dissociation energy

$\mathrm{E}_{4}=$ Morse parameter

$\mathrm{f}=$ intermolecular force

$\mathrm{g}_{1}=$ degeneracy

$\mathrm{H}=$ Hamiltonian

$\mathrm{k}=$ Boltzmann constant

$\mathrm{L}=$ Lagrangian

$\mathrm{m}=$ atomic mass

$\mathrm{n}=$ rate constant

$\mathrm{N}=$ number of molecules

$\mathrm{N}^{*}=$ number of molecules in a particular energy level

$\mathrm{p}=$ momentum $\mathrm{q}=$ generalized coordinates

$\mathrm{r}=$ position vector

$\mathrm{r}_{\mathrm{e}}=$ mean bond length

$r_{i j}=$ position vector between atoms $i$ and $j$

$\mathrm{t}=$ time

$\mathrm{T}=$ temperature

$\mathrm{u}=$ intermolecular potential

$\mathrm{v}=$ velocity vector

$\mathrm{V}=$ intramolecular potential

$\alpha=$ degree of dissociation

$\beta=$ Morse parameter

$\varepsilon=$ Lennard-Jones energy parameter

$\varepsilon_{\mathrm{v}}=$ vibrational energy

$v=$ quantum energy level

$\theta_{\mathrm{d}}=$ characteristic dissociation temperature

$\rho=$ density

$\rho_{\mathrm{d}}=$ characteristic density

$\sigma=$ Lennard-Jones size parameter

$\omega_{\mathrm{e}}=$ spacing of vibrational energy levels

$\mathrm{x}_{\mathrm{e}} \omega_{\mathrm{e}}=$ first anharmonic correction

\section{$\underline{\text { Introduction }}$}

The ability to model a combustion process numerically is very important to the development of future propulsion systems. For example, a computer model would be useful in cases where an analytical solution was not valid or an experiment was too difficult to perform. One method of modeling chemical reactions through computer simulation is molecular dynamics.

The first step in simulating a reacting gas by molecular dynamics is determining how vibrational energy is distributed among the atoms. Once this is accomplished, modeling simple reactions is possible. One of the least difficult reactions to model is the dissociation and recombination of a homonuclear diatomic species such as hydrogen and oxygen.

\section{Molecular Dynamics}

Molecular dynamics is the numerical simulation of atomic and molecular motions and forces in a system. By observing the trajectories of these particles, many thermodynamic and fluid mechanical properties can be 
calculated. The main advantage of this technique is that its accuracy is only dependent on the selection of an intermolecular potential and the precision of the numerical integrator. No physical properties need to be known nor assumptions made about the simulated medium. However, the disadvantage is that the size of the molecular systems must be extremely small. This is due to the large number of force calculations that must be made.

Modeling the atomic potentials is one of the most important parts of a molecular dynamics simulation. This particular investigation deals with atoms that are free to vibrate within the molecules. Such a system requires both intermolecular and intramolecular potentials. The intramolecular potential is utilized in computing the forces between two atoms of the same molecule, while the intermolecular potential is used to calculate forces between atoms of different molecules.

\section{$\underline{\text { Intermolecular Potential }}$}

In molecular dynamics, the potential is taken to be pairwise additive. This means that the total energy in the system is a sum of the isolated two-body contributions. Three body and higher terms are usually neglected because of the vast increase in computational time and their relatively small influence on gaseous systems. The most commonly used pairwise additive potential, $\mathrm{u}$, for modeling intermolecular forces between atoms separated by a distance $r_{i j}$ is the one proposed by Lennard-Jones. ${ }^{1}$

$$
u\left(r_{i j}\right)=4 \varepsilon\left[\left(\frac{\sigma}{r_{i j}}\right)^{12}-\left(\frac{\sigma}{r_{i j}}\right)^{6}\right]
$$

This potential produces a short range atomic repulsion which simulates the overlap of electron clouds and a long range attraction due to induced dipoles. The only adjustable parameters are a size parameter, $\sigma$, and an energy parameter, $\varepsilon$. Both of these values are a function of the species being simulated. Since the intermolecular forces are necessarily conservative, the force resulting from the above potential is

$$
f(r)=-\frac{d u(r)}{d r}=24 \frac{\varepsilon}{\sigma}\left[2\left(\frac{\sigma}{r}\right)^{13}-\left(\frac{\sigma}{r}\right)^{7}\right]
$$

\section{$\underline{\text { Intramolecular Potential }}$}

The potential between two atoms of the same molecule differs slightly from the intermolecular potential. In order to conform to spectroscopic experiments, the intramolecular potential is required to have a potential well at the mean bond length and approach the dissociation energy as the atoms become infinitely separated. Also, like the Lennard-Jones potential, it must go to infinity as the atoms approach each other. One such model that conforms to these specifications is the Morse potential. ${ }^{2}$ This potential is given by

$$
V(r)=D e^{-2 \beta\left(r-r_{e}\right)}-2 D e^{-\beta\left(r-r_{e}\right)}+E_{\infty}
$$

where $\mathrm{D}$ is the dissociation energy, $\mathrm{r}$ is the internuclear separation, $r_{e}$ is the mean bond length and $\beta$ and $E_{\infty}$ are constants computed from spectroscopic data. Unlike Lennard-Jones, the Morse potential is based on four parameters. The differences between the two potentials can be seen in Figure 1. This figure shows that the depth of the Morse potential well compared to its long range potential is far greater than that of Lennard-Jones. In addition, the Morse potential permits smaller separation distances between the atoms.

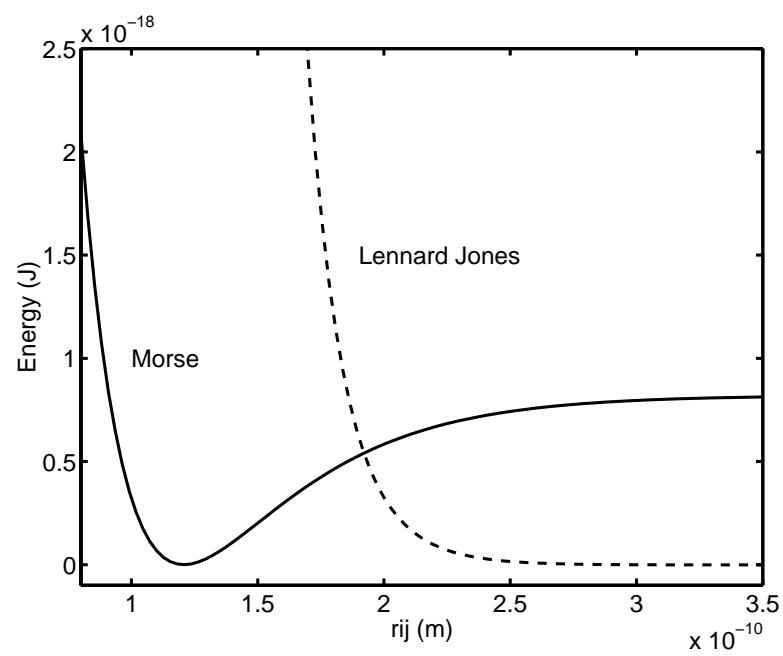

Figure 1. Morse and Lennard-Jones Potentials

\section{$\underline{\text { Finite Difference Method }}$}

Once the potentials have been established, the equations of motion can be produced. One typical form of the equations of motion in molecular dynamics is the Hamiltonian. ${ }^{3}$ The Hamiltonian is defined in terms of the Lagrangian $\mathrm{L}$ and a set of generalized coordinates $\mathrm{q}$

$$
H(\vec{p}, \vec{q})=\sum_{k} \dot{q}_{k} p_{k}-L(\vec{q}, \dot{\vec{q}})
$$

where $\mathrm{p}$ is the momentum and is given by 


$$
p_{k}=\frac{\partial L}{\partial \dot{q}_{k}}
$$

and

$$
\dot{q}_{k}=\frac{\partial H}{\partial p_{k}} \quad \dot{p}_{k}=-\frac{\partial H}{\partial q_{k}}
$$

In terms of Cartesian coordinates, the equations of motion become

$$
\dot{\vec{r}}_{i}=\frac{\vec{p}_{i}}{m_{i}} \quad \dot{\vec{p}}_{i}=-\nabla u=\vec{f}_{i}
$$

These equation of motion must now be integrated to obtain the trajectories of the atoms. One of the most common finite difference methods is the velocity Verlet algorithm. ${ }^{4}$ This technique has the advantage of being third-order accurate even though it contains no thirdorder derivatives. The velocity Verlet is a two step process. First, the position is advanced one time step and the velocity is advanced half of a time step.

$$
\begin{gathered}
r(t+\Delta t)=r(t)+v(t) \Delta t+\frac{\Delta t^{2}}{2} a(t) \\
v\left(t+\frac{\Delta t}{2}\right)=v(t)+\frac{\Delta t}{2} a(t)
\end{gathered}
$$

At this point, the forces are computed and velocity is advanced another half time step.

$$
v(t+\Delta t)=v\left(t+\frac{\Delta t}{2}\right)+\frac{\Delta t}{2} a(t+\Delta t)
$$

This method offers simplicity and good stability for a relatively large time step. The velocity Verlet algorithm also has the advantage that it is relatively easy to program and requires little computer memory. These qualities are essential when modeling large numbers of atoms.

\section{Vibrational Energy Distribution}

The first goal of this molecular dynamics simulation was to model the vibrational energy distribution within a system of oxygen molecules. Vibrational energy is distributed in discrete energy levels according to the Boltzmann distribution. ${ }^{5}$

$$
\frac{N^{\prime *}}{N}=\frac{g_{l} e^{-\varepsilon_{v} / k T}}{\sum g_{l} e^{-\varepsilon_{v} / k T}}
$$

where $N^{\prime} * / \mathrm{N}$ is the fraction of molecules in a particular level, $\mathrm{g}_{\mathrm{l}}$ is the degeneracy, $\varepsilon_{\mathrm{v}}$ is the vibrational energy, $\mathrm{k}$ is the Boltzmann constant and $\mathrm{T}$ is temperature. For vibrational energy distribution, there is no degeneracy. This means that there is only one state per level or $\mathrm{g}_{\mathrm{l}}$ is unity.

However, since vibrational energy increases in discrete increments, a relationship must be formed between the quantum energy levels and the vibrational energy. This relationship is given by

$$
\varepsilon_{v}=\omega_{e}(v+.5)-x_{e} \omega_{e}(v+.5)^{2}-.5 \omega_{e}+.25 x_{e} \omega_{e}
$$

where $v$ is the quantum energy level, $\omega_{\mathrm{e}}$ is the spacing of the vibrational energy levels, and $x_{e} \omega_{e}$ is the first anharmonic correction. ${ }^{6}$

In contrast, the molecular dynamics code outputs vibrational energy which must then be converted to discrete quantum levels by solving equation 14 for $v$. This operation is performed on every molecule at every time step. One further complication is that $v$ must be an integer. The most efficient method to convert $v$ is simply to truncate it. Truncation ensures that the zero energy level bin is equal in size to the other bins. The last step is to count the number of molecules in each energy level. At this point the results can be compared to kinetic theory.

\section{$\underline{\text { Reactions }}$}

The second part of this investigation focuses on modeling simple chemical reactions using molecular dynamics. The first step is to simulate the dissociation and recombination of a single molecular species, in this case, hydrogen and oxygen. Then, the complexity is increased by running a simulation that contains both hydrogen and oxygen and observing the reactions that occur in this dual species system.

\section{Kinetic Theory}

According to kinetic theory, dissociation occurs when a second body impacts a molecule at a sufficient energy level. In the case of a single species system, only one type of atom is present, which means the second body can be either another molecule or another atom. If the impact occurs with an energy greater than the dissociation energy of the molecule, the molecule dissociates.

Similarly, recombination is modeled as a three body reaction. The first two bodies are atoms, while the third 
is another atom or molecule. When the three bodies collide, energy is released as the two atoms combine. This energy is then transferred to the third body in the form of translational motion. This type of reaction is less common than dissociation because it involves the collision of three bodies. Hence, an equation can be formed to describe the degree of dissociation in a system of diatomic molecules as a function of time. ${ }^{5}$

$$
\frac{d \alpha}{d t}=\frac{C_{f}}{m} T^{n} \rho\left[(1-\alpha) e^{-\theta_{d} / T}-\frac{\rho}{\rho_{d}} \alpha^{2}\right]
$$

In this equation, $\mathrm{C}_{\mathrm{f}}$ and $\mathrm{n}$ are experimentally determined rate constants. In addition, a similar equation can be formed for the equilibrium degree of dissociation.

$$
\begin{aligned}
& \frac{\alpha^{* 2}}{1-\alpha^{*}}=\frac{e^{-\Theta_{d} / T}}{\rho} . \\
& {\left[m\left(\frac{\pi m k}{h^{2}}\right)^{3 / 2} \Theta_{r} \sqrt{T}\left(1-e^{-\Theta_{v} / T}\right) \frac{\left(Q_{e l}^{a}\right)^{2}}{Q_{e l}^{a a}}\right]}
\end{aligned}
$$

In this equation, $\alpha^{*}$ is the equilibrium degree of dissociation, $\Theta_{\mathrm{d}}$ is the characteristic temperature of dissociation, $\mathrm{T}$ is the temperature, $\rho$ is the density, $\mathrm{m}$ is the atomic mass, $\mathrm{k}$ is the Boltzmann constant, $\mathrm{h}$ is the Planck constant, $\Theta_{\mathrm{r}}$ is the rotational characteristic temperature, $\Theta_{v}$ is the vibrational characteristic temperature, $\mathrm{Q}_{\mathrm{el}}{ }^{\mathrm{a}}$ is the atomic electronic partition function, and $\mathrm{Q}_{\mathrm{el}}{ }^{\mathrm{aa}}$ is the molecular electronic partition function. However, this is a very complicated expression and can be simplified by the Lighthill approximation. ${ }^{5}$ Lighthill found that the value of the bracketed expression remained relatively constant and could be given by $\rho_{d}$, the characteristic density. This simplifies Eq. 16 to the form.

$$
\frac{\alpha^{* 2}}{1-\alpha *}=\frac{e^{-\Theta_{d} / T}}{\rho} \rho_{d}
$$

\section{$\underline{\text { Molecular Dynamics }}$}

Unfortunately, it is not possible to apply this technique to a molecular dynamics simulation that uses atomic potentials because there are no discrete collisions. The distance between atoms never actually goes to zero because this would cause an infinite repulsive potential. Therefore, another technique must be used to simulate dissociation and recombination reactions.
The method chosen for this molecular dynamics code was 'curve hopping'. $7,8,9$ Dissociation takes place when the potential between two atoms of an oxygen molecule exceeds some critical value. The point at which this occurs is relatively ambiguous because it is difficult to explicitly define the point on the Morse potential curve where the molecule dissociates. Therefore, in this simulation, a molecule is said to dissociate when the potential between the two atoms reaches $96 \%$ of the dissociation energy. This value was chosen because it produced suitable agreement with kinetic theory. However, the results were only slightly affected by varying this ratio between $90 \%$ and $99 \%$. As dissociation occurs, the atoms jump from the Morse to the Lennard-Jones potential, hence 'curve hopping'.

Recombination of atoms is performed in the opposite manner. When the potential between two dissociated atoms decreases below $0.5 \mathrm{kT}$, the atoms combine to form an oxygen molecule. ${ }^{8}$ When recombination occurs the potential between the two atoms is switched from the Lennard-Jones to the Morse potential.

Both recombination and dissociation require a change of potential surface for the two atoms involved in the process. When this switch occurs, there is a jump in the potential energy of the constituent atoms. To account for this, the atoms' velocities are scaled to adjust their kinetic energy to match the change in potential so the total energy of the system remains constant. ${ }^{7}$ Subsequently, as the atoms move with their new velocities, they affect their neighbors. In the case of recombination, the energy released by the creation of an oxygen molecule is transferred to the surrounding atoms by this process. Therefore, no third body is required for recombination to occur.

\section{$\underline{\text { Molecular Dynamics Simulation }}$}

Two different types of codes are used in this molecular dynamics simulation. The first is a vibrational energy distribution code which only models atomic vibration and does not allow the molecules to dissociate. This program is used to compute the Boltzmann distribution. The second code is a reaction code which is very similar to the vibrational energy distribution program. The only major difference is the reaction code allows dissociation and recombination. This is accomplished by updating an array that specifies which atoms are connected. The reaction code is used to compute the degree and rate of dissociation in the system. Both hydrogen and oxygen were simulated individually by the two codes and a reaction simulation containing both hydrogen and oxygen was performed.

In each case, the simulation begins with the entire system as molecular species and arranged in a facecentered cubic lattice. A cubical environment is utilized 
and periodic boundary conditions are imposed. When computing the vibrational energy distribution, 256 molecules were used. However, only 108 molecules were present in the single species reaction simulation. This reduction in atoms vastly increased the speed of the code which was necessary because of the relatively long time period required for the system to achieve equilibrium. Finally, the dual species reaction code used 128 oxygen and 128 hydrogen molecules in order to achieve high resolution data. All of these codes use a 1 fs time step for oxygen and a 0.2 fs time step for hydrogen. Such small time steps are required to accurately model the high frequency vibrations of the atoms. Standard molecular dynamics codes use time steps an order of magnitude larger.

In addition, these programs employ a variety of techniques which greatly increase the performance of the code. The first of these methods reduces the number of force calculations by limiting the range of the Lennard-Jones potential. This technique is valid because the intermolecular potential becomes very small at atomic separation distances of only a few diameters. For this simulation, a cut-off radius of $2.5 \sigma$ was selected. There is a resulting jump in potential energy as an atom crosses this radius, but it is usually very small. Also, since the force that atom 1 exerts on atom 2 is equal to the force that atom 2 exerts on atom 1 , only one force calculation needs to be performed for both situations.

Four identical versions of the vibrational energy distribution code were submitted to four processors of COCOA. The temperature and density of the systems would be the same, but the initial velocities of the atoms were chosen at random. This caused each case to produce slightly different results that when averaged reduced fluctuations in the vibrational energy distribution curve. COCOA is a collection of 25 dual processor Pentium ${ }^{\circledR}$ II computers linked together by fast Ethernet. ${ }^{10}$ A 20,000 time step run would typically take about 2 hours to complete. In the case of the reacting gas, eight identical codes were executed on COCOA with varying initial conditions. In the case of hydrogen, 45 million time steps were used. This code would take about 27 hours to run. In the case of oxygen, 70 million time steps were used, which took about 48 hours to run. This means that the hydrogen code ran at 1.67 million time steps per hour, while the oxygen code ran at 1.45 million time steps per hour. The difference between the two codes was the number of linked list cells used. In the case of hydrogen, the density of the system allowed 9 cells per dimension to be used, while the oxygen case only allowed 8 cells per dimension. This is a perfect example of the effect of linked list cells on the performance of a molecular dynamics code.

\section{$\underline{\text { Results and Discussion }}$}

The first goal of this investigation was to determine the vibrational energy distribution in a system of molecules. Simulations were run at a variety of temperatures using both hydrogen and oxygen. In all cases, 256 molecules were present and the code was executed for 20,000 time steps. Since the distribution is averaged over time, the more time steps performed, the smaller the statistical fluctuations.

Figures 2 and 3 show the vibrational energy distribution of oxygen calculated using molecular dynamics compared to the Boltzmann distribution computed for both harmonic and anharmonic interatomic potentials at a temperature of $1942 \mathrm{~K}$. They show excellent agreement between the molecular dynamics code and the Boltzmann distribution. These plots are given in terms of the normalized fraction of molecules relative to the zero vibrational energy level. Since this molecular dynamics simulation utilizes the Morse potential, the computational points should lie on the anharmonic distribution. The logarithmically plotted data points show a small deviation from the theoretical curve at higher energy levels. This data could be improved by increasing the number of time steps and increasing the number of molecules in the system.

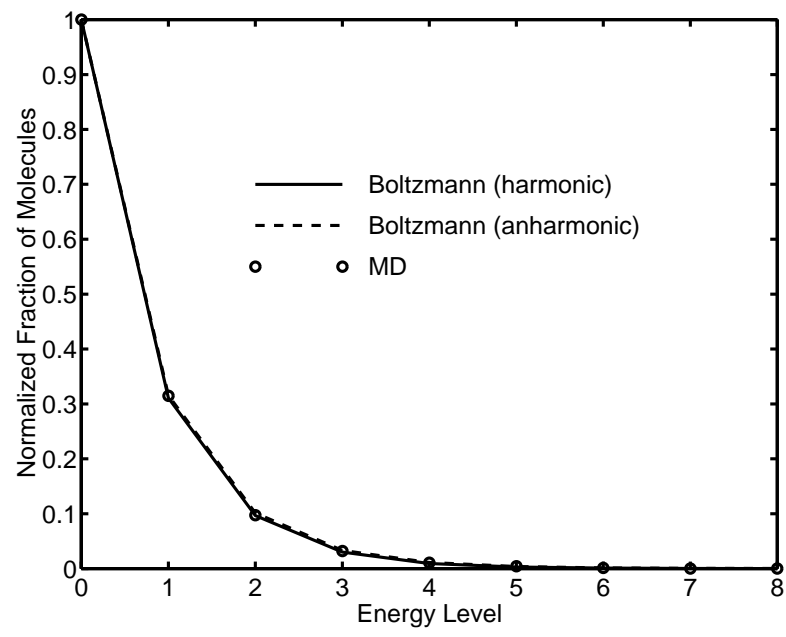

Figure 2. Vibrational energy distribution of oxygen molecules calculated using molecular dynamics compared to the Boltzmann distribution for both harmonic and anharmonic interatomic potentials $\mathrm{T}=1942 \mathrm{~K}$ 


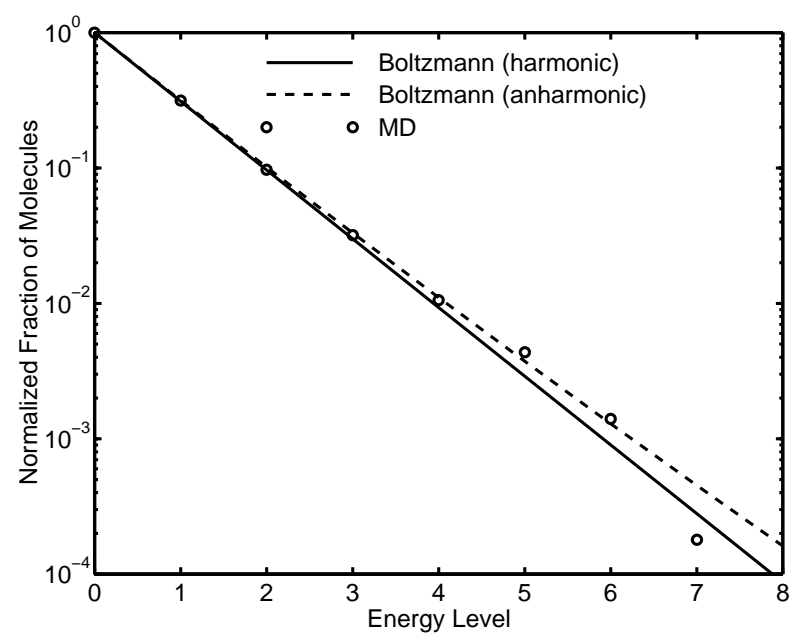

Figure 3. Vibrational energy distribution of oxygen molecules calculated using molecular dynamics compared to the Boltzmann distribution for both harmonic and anharmonic interatomic potentials $\mathrm{T}=1942 \mathrm{~K}$

Figures 4 and 5 show the vibrational energy distribution of hydrogen molecules calculated using molecular dynamics compared to the Boltzmann distribution computed for both harmonic and anharmonic interatomic potentials at a temperature of 3935 K. Excellent agreement can be seen between the molecular dynamics results and the Boltzmann distribution. However, the molecular dynamics data appear to lie on the harmonic Boltzmann distribution instead of the anharmonic distribution. This is unexpected because the Morse potential, upon with this simulation is based, is an anharmonic potential. Also, as indicated by the high temperature at which this simulation was run, hydrogen molecules require much greater energy to reach higher energy levels. It is also interesting to note the greater divergence in the theoretical harmonic and anharmonic distributions compared to those of oxygen.

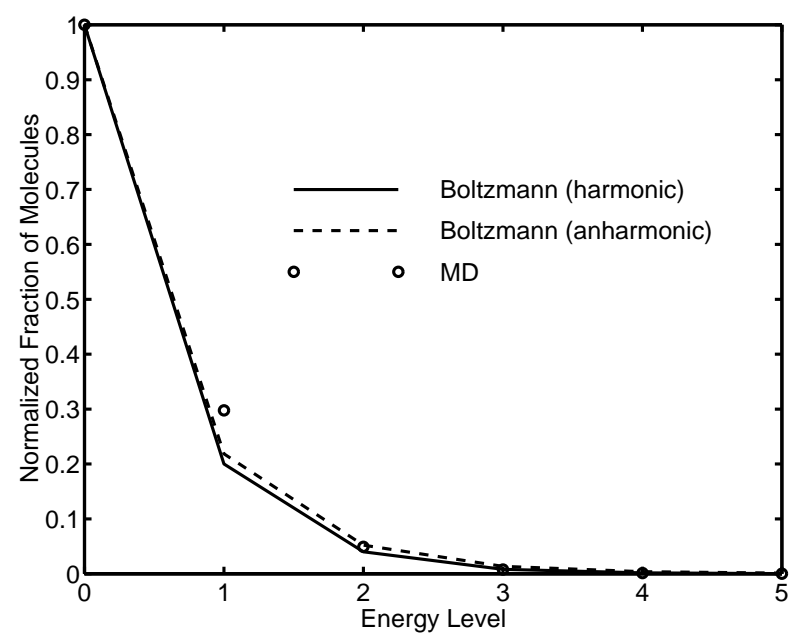

Figure 4. Vibrational energy distribution of hydrogen molecules calculated using molecular dynamics compared to the Boltzmann distribution for both harmonic and anharmonic interatomic potentials $\mathrm{T}=3935 \mathrm{~K}$

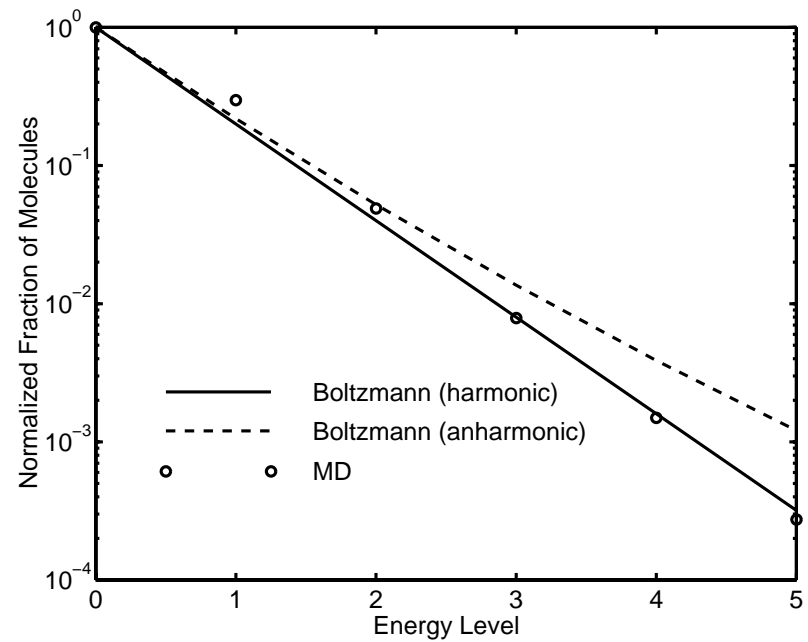

Figure 5. Vibrational energy distribution of hydrogen molecules calculated using molecular dynamics compared to the Boltzmann distribution for both harmonic and anharmonic interatomic potentials $\mathrm{T}=3935 \mathrm{~K}$

The second part of this investigation examined the dissociation and recombination reactions in a system of atomic and molecular species. Due to the large number of time steps required, only 108 molecules were used. In addition, only high pressure and high temperature cases were examined in order to minimize the time required for the system to achieve equilibrium. Figure 6 shows the molecular dynamics simulation the degree of dissociation of hydrogen compared to kinetic theory. 


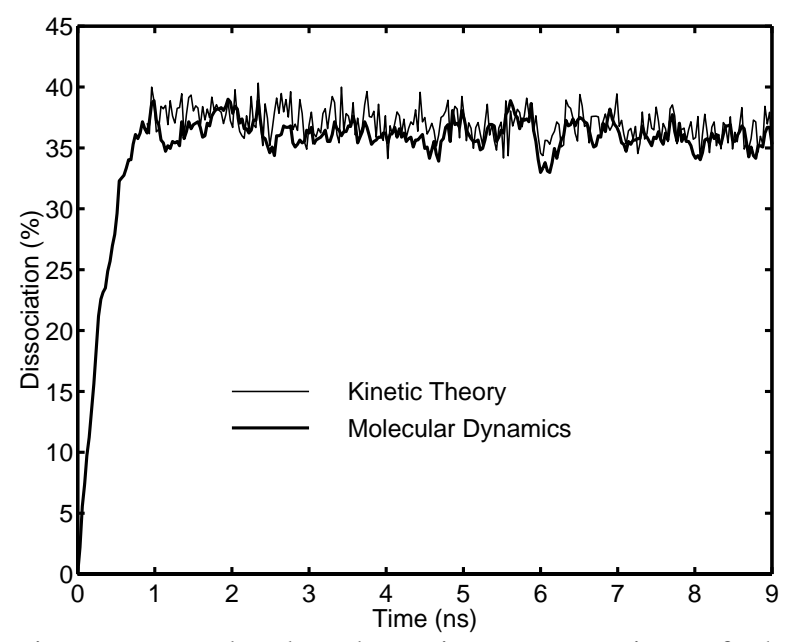

Figure 6. Molecular dynamics computation of the degree of dissociation of hydrogen compared to kinetic theory

In this plot, the blue line represents the molecular dynamics data, while the red line represents kinetic theory. As can be seen from the plot, the molecular dynamics simulation begins with no dissociation. The system reaches equilibrium quickly and is then compared to equilibrium kinetic theory. The kinetic theory fluctuates based on fluctuations in temperature. The Lennard-Jones parameters chosen for this simulation were $\sigma=2.7 \mathrm{E}-10 \mathrm{~m}$ and $\varepsilon=4.0 \mathrm{E}-22 \mathrm{~J}$. This plot demonstrates good agreement between molecular dynamics simulation and kinetic theory.

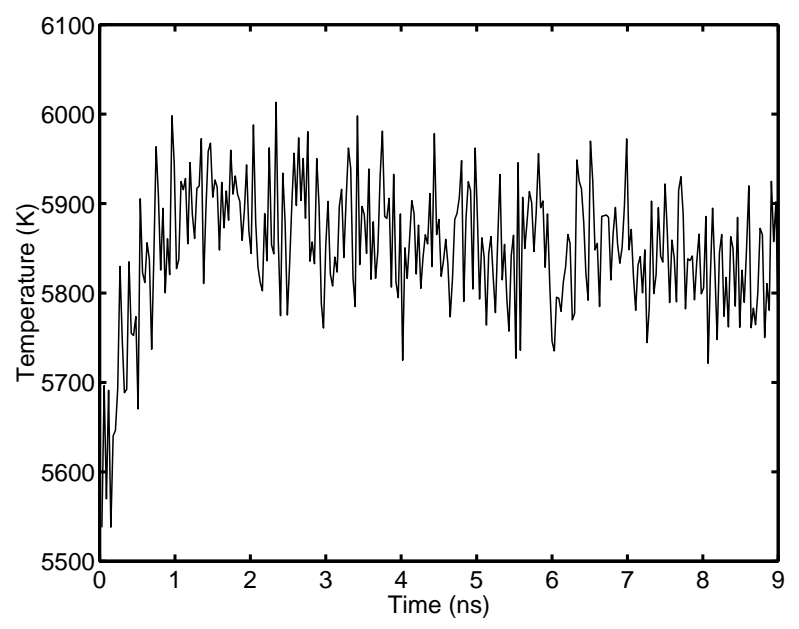

Figure 7. Temperature fluctuations during the simulation

Figure 7 shows the temperature fluctuations that occur during the simulation. This plot clearly shows the initial rise in temperature that occurs when the molecules are dissociating. This temperature rise is caused by the breaking of chemical bond. When a bond breaks, the chemical potential energy of the bond is converted to kinetic energy in the form of increased atomic motion. This naturally, increases the temperature of the system. It is also interesting to see the high frequency fluctuations in temperature that occur when the system reaches equilibrium. The temperature can vary by up to $200 \mathrm{~K}$. This temperature fluctuation is caused by the large number of molecules that are dissociating and recombining.

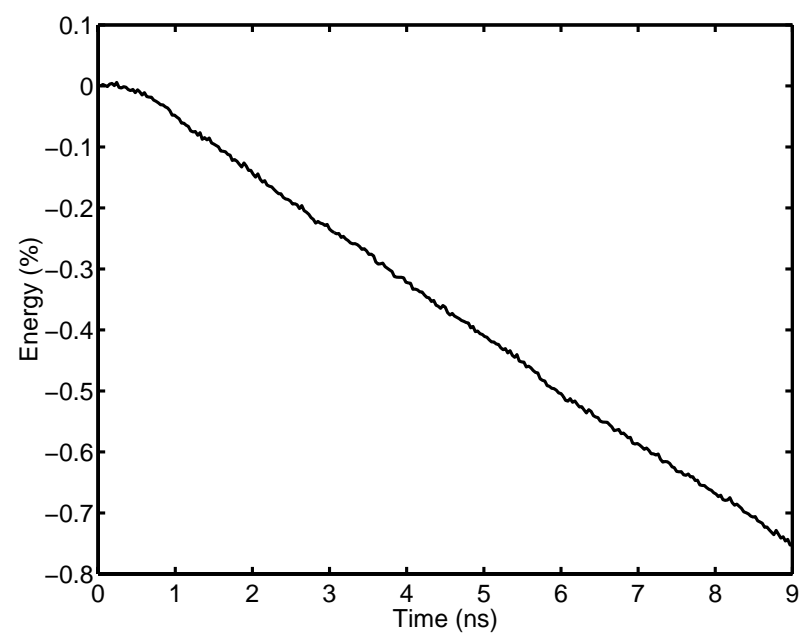

Figure 8. Energy drop during the simulation

Figure 8 shows the energy drop that occurs during the simulation. The drop in energy takes place over a relatively long span of 45 million time steps. In this period, the energy drops by roughly $3 / 4$ of a percent. This is not a significant loss in energy and does not appear to adversely affect the system. However, this loss of energy cannot be remedied because of the nature of the dissociation code. When a molecule dissociates the energy released is transferred into the resulting atoms. This increase in kinetic energy causes the atoms to dramatically increase their velocity. Frequently, the velocity of the atoms exceeds the maximum value allowed by the time step. The result is a loss of total energy in the system. This problem could be solved by decreasing the time step, but that would cause the code to run slower.

In addition to the hydrogen simulations, molecular dynamics simulation of the dissociation of oxygen was performed. Similar to the hydrogen simulations, three cases were run at various temperatures to observe how the oxygen Lennard-Jones parameters varied. Furthermore, since oxygen is one of the main components of the atmosphere, its dissociation has been more closely examined by experimenters. This means that rate of dissociation data can be located. Therefore, the molecular dynamics code can not only be compared to the theoretical equilibrium degree of dissociation, but also to the kinetic theory rate equation. The LennardJones parameters chosen for this run were $\sigma=3.0 \mathrm{E}-10$ 
$\mathrm{m}$ and $\varepsilon=9.0 \mathrm{E}-22 \mathrm{~J}$. Figure 9 shows the molecular dynamics results compared to the equilibrium degree of dissociation, while Figure 10 shows the molecular dynamics results compared to the kinetic theory rate equation.

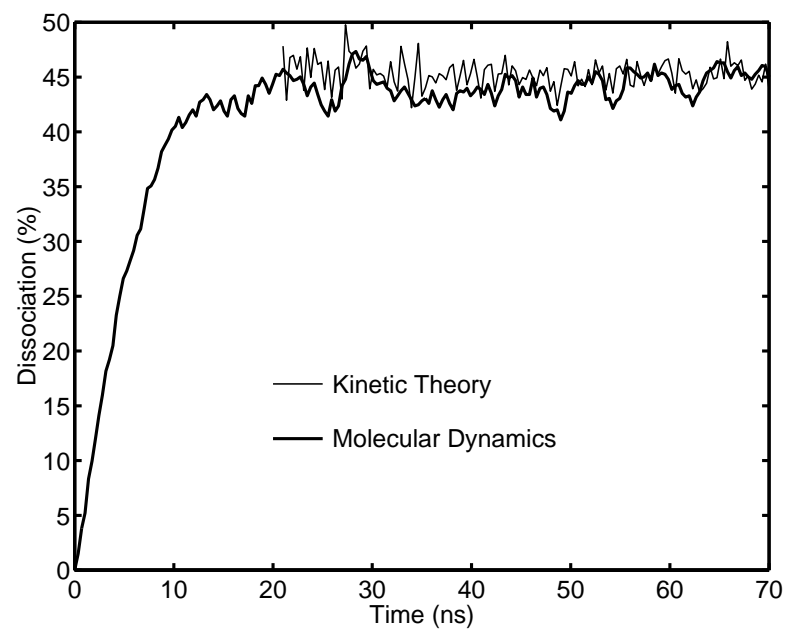

Figure 9. Molecular dynamics computation of the degree of dissociation of oxygen compared to kinetic theory

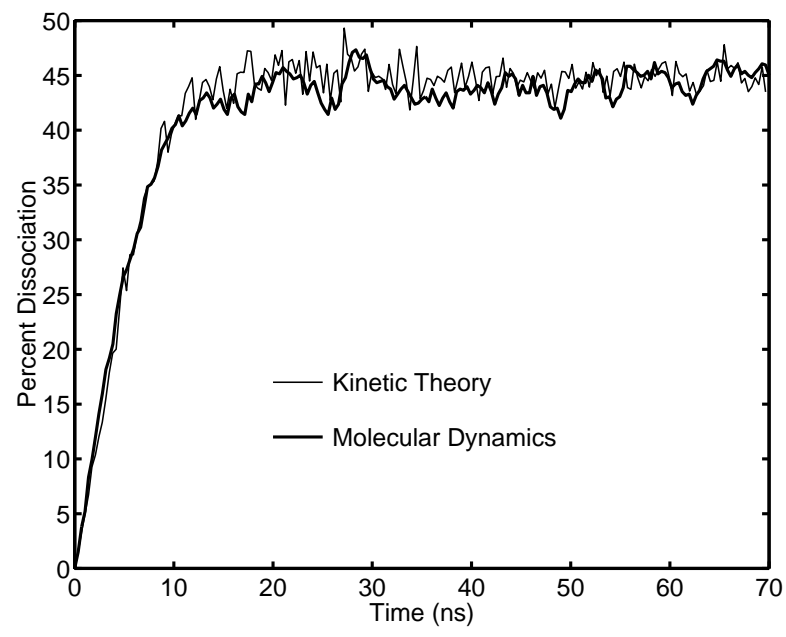

Figure 10. Molecular dynamics computation of the degree of dissociation of oxygen compared to kinetic theory

Again there is excellent agreement between the molecular dynamics results and kinetic theory. Just as in the hydrogen runs, there is an initial temperature rise as dissociation occurs. However, the system of oxygen takes longer to reach equilibrium.

According to kinetic theory, Eq. 15, the rate of dissociation is based on two parameters $C_{f}$ and $n$. $n$ is very difficult to measure experimentally due to the limitations of the test apparatus, principally shock tubes. $^{11}$ Therefore, most experimentalists arbitrarily chose $\mathrm{n}$ to be -1.5 for oxygen and experimentally determine $\mathrm{C}_{\mathrm{f}}$. In addition, $\mathrm{C}_{\mathrm{f}}$ can vary up to $50 \%$. Therefore, the experimentally determined $\mathrm{C}_{\mathrm{f}}$ was used $^{12}, 1.9 \mathrm{E} 21$, and $\mathrm{n}$ was chosen to correspond to the molecular dynamics data, -1.6. However, there is very little difference between this value and the one arbitrarily selected for experiments.

\section{$\underline{\text { Conclusions }}$}

Vibrational energy distribution and dissociation reactions of hydrogen and oxygen were examined by molecular dynamics simulations. Results of the vibrational energy distribution studies have shown excellent agreement with the Boltzmann distribution. Both the rate of dissociation and the equilibrium degree of dissociation have also compared well to kinetic theory. This investigation has demonstrated that simple large-scale chemical reactions can be modeled by molecular dynamics.

However, this project is only the first step in modeling chemical reactions by the 'curve hopping' method of molecular dynamics. The simulations performed in this investigation only contained a few hundred atoms. Future simulations should be considerably larger. This could be accomplished without diminished performance by parallelization of the code. In addition, water, which was not modeled in this program should be included in future simulations. By including water, the hydrogen - oxygen combustion process can be completely modeled by molecular dynamics. Finally, the combustion of hydrocarbons should be simulated. Due to the large number and different type of atoms in even the simplest hydrocarbon, the complexity of the system will be greatly increased.

\section{$\underline{\text { References }}$}

${ }^{1}$ Rapaport, D. C. The Art of Molecular Dynamics Simulation. Cambridge University Press, $1^{\text {st }}$ edition, 1995.

${ }^{2}$ Morse, P. M. Diatomic molecules according to the wave mechanics. II. Vibrational levels. Physical Review, 34, pp. 57-64, 1929.

${ }^{3}$ Haile, J. M. Molecular Dynamics Simulation: Elementary Methods. John Wiley, New York, $1^{\text {st }}$ edition, 1992.

${ }^{4}$ Allen, M. P., and Tildesley, D. J. Computer Simulation of Liquids. Clarendon Press, Oxford, $1^{\text {st }}$ edition, 1987. 
${ }^{5}$ Vincenti, W. G., and Kruger, C. H. Introduction to Physical Gas Dynamics. Krieger Publishing Company, Malabar, Florida, 1986.

${ }^{6}$ Suchard, S. N., and Melzer, J. E. Spectroscopic Data, Volume 2: Homonuclear Diatomic Molecules. The Aerospace Corporation, Los Angeles, CA, 1976.

${ }^{7}$ Liu, Q., Wang, J., and Zewail, A. H. Solvation ultrafast dynamics of rections. 10. Molecular dynamics studies of dissociation, recombination and coherence. Journal of Physical Chemistry, 99, pp. 11321-11332, 1995.

${ }^{8}$ Song, T. T., Hwang, Y. S., and Su, T. M. Recombination reactions of atomic chlorine in compressed gases. 3. Molecular dynamics and Smoluchowski equation studies with argon pressure up to 6kbar. Journal of Physical Chemistry, 101, pp. 3860-3870, 1997.

${ }^{9}$ Amar, F. G., and Berne, B. J. Reaction dynamics and the cage effect in microclusters of $\mathrm{Br}_{2} \mathrm{Ar}_{\mathrm{n}}$. Journal of Physical Chemistry, 88, pp. 6720-6727, 1984.

${ }^{10}$ Modi, A. COst effective COmputing Array. http://cocoa.ihpca.psu.edu, 1998.

${ }^{11}$ Park, C. Two-temperature interpretation of dissociation rate data for $\mathrm{N}_{2}$ and $\mathrm{O}_{2}$. AIAA paper 880458, January 1988.

${ }^{12}$ Byron, S. R. Measurement of the rate of dissociation of oxygen. Journal of Chemical Physics, 30(6), pp. 1380, 1959. 